\title{
The Research of the Driver Attention Field Modeling
}

\author{
Pengfei Tao, ${ }^{1,2}$ Hongyu Hu, ${ }^{2}$ Zhenhai Gao, ${ }^{1}$ Xin Liu, ${ }^{3,4}$ Xianmin Song, ${ }^{2}$ Yan Xing, ${ }^{2}$ \\ Yuzhou Duan, ${ }^{2}$ and Fulu Wei ${ }^{2}$ \\ ${ }^{1}$ State Key Laboratory of Automotive Simulation and Control, Jilin University, Changchun 130022, China \\ ${ }^{2}$ College of Transportation, Jilin University, Changchun 130022, China \\ ${ }^{3}$ School of Management, Jilin University, Changchun 130022, China \\ ${ }^{4}$ Network Center, Jilin University, Changchun 130022, China
}

Correspondence should be addressed to Hongyu Hu; huhongyu@jlu.edu.cn

Received 28 September 2013; Accepted 4 December 2013; Published 6 January 2014

Academic Editor: Huimin Niu

Copyright (C) 2014 Pengfei Tao et al. This is an open access article distributed under the Creative Commons Attribution License, which permits unrestricted use, distribution, and reproduction in any medium, provided the original work is properly cited.

For expanding the application scope of car-following, based on the basic idea of the noncontact interaction of the objects in physics, establish an attention field model to describe the driving behavior. Firstly, propose the time distance concept to describe the degree of driver perception to the front one-dimensional space and extend its application range to the two-dimensional space. Secondly, connect the point which has the same time distance to constitute the equipotential line of drivers' attention field equipotent, and establish a model to describe it. Thirdly, define the effective range of the driver's psychological field with the feature of the driver's visual distance range increasing and the angle decreasing. Finally, design the calculation method to collect projection of the object in the psychological field scope and calculate the curve points to determine the object's intensity of psychological field. Preliminarily build the driving behavior model and use the numerical simulation method to simulate the simple transport scenarios; initially verify the validity of the model.

\section{Introduction}

The research on the driving behavior has been the focus in the traffic engineering study. The driving behaviors model establishes a link between the characteristics of the single vehicle and the traffic. It explains the macrotransport phenomena from the moving of the microindividual and makes the development of the traffic flow theory. The driving behaviors model has important applications in the field of traffic simulation, traffic safety, and intelligent driving.

The car-following model is the main form of the model driving behavior. Car-following model was used to describe the driving behavior of following vehicle facing the actions of the leading vehicle in the single lane with limit of overtake. It played an important role in the traffic theory and was widely used in the fields of microscopic traffic simulation, smart driving, traffic safety, and so forth. The researches of car-following have been lasting for years and made a series of results [1-3], and the GM car-following model was proposed firstly and considered the most classic model in those research results $[4,5]$. This model regarded the difference between the leading and following car as the source of stimulation and combined with the space headway and velocity to obtain the driver's acceleration decision making [6-9]. A variety of car model were from derived; the GM modeling idea [10-13]. Lee proposed a GM model with memory effect; the basic idea was that the driver react relied on the stimulation of a period of time rather than the pointin-time. Herman established a model to describe the impact on driver form the multivehicle in the front; it considered that the driver received the effect not only from the single leading car but also from the continuous traffic flow before the him.

With the developing of microscopic traffic simulation technology, the microdriving behavior of single vehicle is focused on $[14,15]$. The models become complex more and more and tend to descript the specific behavior for different conditions to make the simulation realistic. And basing on it, obtain the macrotraffic situation by simulating a number 
of running individual vehicles. The typical achievements contain mental-physical model, fuzzy inference model, safe distance model, and so forth.

A fundamental assumption of the traditional carfollowing model is that all vehicles are travelling in the center of the lane. But it is difficult to achieve this ideal state. Gunay (2007) studied the transverse distribution of the vehicle in the lane, it showed the vehicle occurrence frequency decrease from the center to sides in the lane, similar to normal distribution, and exhibited the lateral offset phenomenon which was prevalent. Meanwhile, the traditional car-following model only considered the effect from the leading vehicle but ignored the front vehicle in the adjacent lane, and this would exert a certain extent impact on the driver.

In addition, most traditional car-following models research the model simply based on the movement of the vehicle. The driver is the operator of the vehicle; his perception of traffic environment would determine the state of the vehicle motion. The previous researches mostly focus on the rules of vehicle running trajectory or the certain physiological thresholds as the trigger of driving operation. Relatively lack the considering of driver's characteristic.

This paper proposes a driver attention field for describing the driver's attention distribution, and establishes a driving behavior model framework with considering the drivers' individual characteristics.

\section{Method}

2.1. The Basic Idea of Modeling. Driving behavior is the combined effect of the driving task and the driving environment to the divers' psychological. The driving task encourages the driver to drive toward the goal direction with the desired speed and leads to a promote effect for the driver's psychological. This impact would change with the characteristics and the motion of the divers. For example, if the velocity is low, the psychological impact would be much more than normal and force the driver to speed up. The psychological impact is also related with the driving states.

And the driving environment may prevent the driver from selecting a higher speed and the corresponding movement direction, causing a psychological stress for driver. This psychological stress reflects the driver's different levels attention to the objects in driving environment. It is related to motion state, attribute and position of the object. For example, the driver would pay more attention to the objects in front than lateral. Similarly, the driver would take notice of the objects with high speed and short distance.

Driving behavior is the balance process under the combined effect of psychological driving and pressure. When the psychological drive has more effects than the psychological pressure, the driver would choose accelerating to reduce the psychological drive. On the contrary, the driver would decelerate or steer the driving direction to reduce the psychological stress. The driver's behavior is running under this balancing process.

This paper takes the distribution of driver's attention in space as a field and applies the field theory to describe the behaviors of the driver responce to the surrounding vehicles, obstacles, signs and markings, and so forth. This attention field is different from the field in physical. The generating source of field in physical would influence the objects in field. The generating source of driver attention field is the driver, and any objects in the field would not be impacted. But the driver would react against the environment under the constraints of its own characteristics. It means the driver determines the strength of the impacts from the objects in driving environment and changes his driving velocity or direction to avoid or reduce the impact of the environment.

In this paper, we design the driver's attention filed as a scalar field and reflect the driver's perception of the surrounding traffic environment with the changing of the field intensity value. The field intensity is the function of the field source (driver) relative position; it is only related to the driver's own characteristics and the motion state of his vehicle. For the objects in the driving environment, just as the positive and negative charged particles in an electric field would be completely different effects, the different types and motion would be differently impacted. It needs to be modeled according to the specific circumstances.

There are a number of similar natures between the attention field and physical field, such as the ultradistance effect; the impact results are not only due to the field itself, but also depend on the properties of objects.

But both principle and the nature of the two fields exist essential difference. In the physical field, such as the electrical fields, the objects in the field would be generated by the source of ultra-distance effect, causing the motion state transform. The objects in the field would be generated by the source of ultradistance effect, causing the motion state transform. The attention field exits in the driver's inner perception. It is not able to affect the objects in it and change the states of objects. But the field could transmit the psychological pressure or driving force to the driver. The driver adjusts his own motion (such as speed up or down, change the direction) to mitigate the pressure impacts from objects and achieves the ultradistance effects.

2.2. Establish the Modeling Frame. Analyse the impact of the driver from the driving environment, as Figure 1 shows. The vehicle $n$ is the study object, which exists around an attention field; we also call it target vehicle below. And set the vehicle locate in the field range $n-1$.

The attention field of vehicle $n$ reflects the time the vehicle $n$ reaches a different spatial locations and also shows the perception of vehicle $n$ driver about the threat degree from the object in this locate.

The attention field of vehicle $n$ driver is created by the driver's subjective perspective. The vehicle $n$ driver could not accurately determine the $n-1$ car driver's next move. So the vehicle $n$ driver would adjust his operating status (including velocity and direction) to achieve the ultra-distance effect to himself. The magnitude and direction of the effect are closely related to the attention field intensity of vehicle $n-1$ location and the relative speed between the vehicle $n$ and $n-1$.

It could be more general; expand the vehicle $n-1$ to arbitrary objects, including stationary obstacles, traffic signs, 




FIGURE 1: The attention field schematic.

and so forth. The impact of vehicle $n$ driver could be described by the attention field proposed above.

The basic idea of modeling the attention field is as follow: firstly, base on the judgment of the field distribution in the space, secondly, analyse the distribution characteristics of equipotential lines, and finally, build a specific potential field values to get the field intensity distribution model.

The attention field exists in the target vehicle moving plane. There must be many equipotential lines in it. If the form of the equipotential lines model is ascertained, the specific calculation method could be relatively easily gotten.

In the vehicle traveling direction of a one-dimensional line, the reciprocal of the time distance could express the form of the field intensity function. The time distance refers to the time of the vehicle reaches the specified position with current speed. Obviously, the greater the time distance is, the lower the attention the driver pays to the front position, which means the lower the field intensity.

Obviously, in case of a steady velocity of target vehicle and a unchanging angle between the target vehicle travelling direction and point-target vehicle line, the closer to the target vehicle, the greater attention field intensity is. And in the case of a fixed distance, the bigger the angle between the direction of the target car driving and the point-target vehicle lines, the weaker the field strength is.

As shown in Figure 2, set the point $P_{1}$ along the direction the target vehicle travel direction. The curve contains the point $P_{1}$ and $Q_{1}$ is an arc of a circle with the center point $O$. It is clear that with the same distance to point $O$, the line $O Q_{1}$ has the bigger angle with target traveling direction than $O P_{1}$. According to the previous relationship between field intensity and angle, the field intensity at point $P_{1}$ is stronger than $Q_{1}$. In the same angle, the closer to the target vehicle the bigger the field strength, and there is a point with the same field intensity of $P_{1}$ between the point is $O$ and $Q_{1}$; let it be $P_{2}$. Similarly, deduce $P_{3}, P_{4}$, and many other potential points with the field intensity equal to $P_{2}$. Connect these points and obtain the approximate shape of equipotential lines as Figure 2 shows.

It can be considered that the driver's attention field intensity is the function of the time-distance in the traveling direction.

When close to the target vehicle, the small time distance would produce relatively large psychological pressure

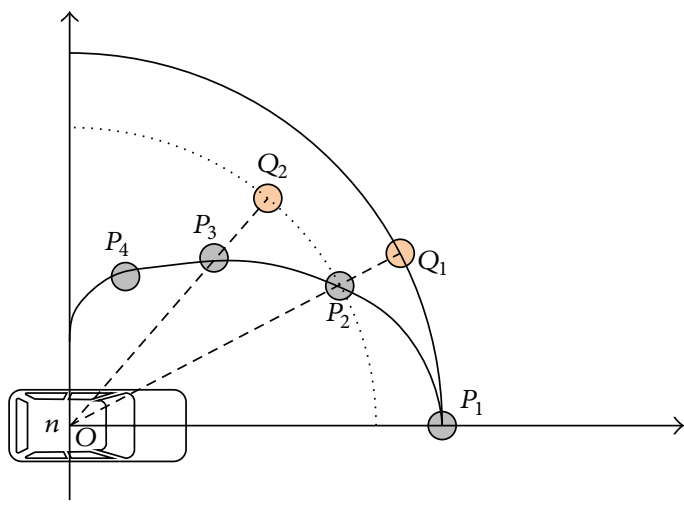

FIGURE 2: The attention field equipotential line shape schematic.

and from a big field intensity. And in the position away from the target vehicle, the driver would reduce his concern degree with the ample response time, and the field intensity is relatively small.

Thus, determine that the field intensity basic form in the traveling direction is the function of the reciprocal of the time-distance as follows:

$$
E b_{n}\left(P_{x}\right)=\frac{v_{n}+v_{\varepsilon}}{L_{0}}
$$

The $P_{x}$ is the point in the target vehicle traveling direction. The $E b_{n}$ is the basic field intensity; three is positive correlation with the field intensity. $v_{n}$ is the velocity of the target vehicle driver in. $L_{0}$ is the distance of between the target and the point $P_{x}, v_{\varepsilon}$ is the correction value.

2.2.1. Establish the Equipotential Lines. Because the field intensity value could be determined by the target vehicle speed and the distance in the traveling direction, choosing a particular point on the traveling line as the reference point and constructing the corresponding equipotential lines according to that point are needed.

Obviously, the shape of the equipotential lines is related to the driver's individual character. The cautious drivers would invest more attention to the space on the besides than aggressive ones. And in addition, the equipotential lines shape also depends on the velocity of target vehicle. In the case of the high speed, the driver would reduce his attention to the besides space, and the shape of the equipotential lines would become flat, and if the vehicle is running with the low speed, compared with the previous case, the equipotential lines shape would be relatively mellow.

For the point in the traveling direction of the target vehicle, after determining the field intensity value of this point, it needs to find the set of points with the same intensity value in the space. Then model according to the general form equipotential lines.

The point in equipotential line with the specific field intensity value would get near the target vehicle with the angle increasing, and when angle is zero, the distance between the target vehicle and the point is biggest. If the point is at the vertical direction of the vehicle, the distance between 




FIgURE 3: The two special points in the attention field equipotential line.

the target vehicle and the point reach is the smallest. There should be a link between the minimum and maximum values.

In Figure 3, the point $P_{y}$ is in the direction vertical to the vehicle travel. The point $P_{x}$ is on the vehicle traveling direction.

If the points are fixed, with the speed of target vehicle $n$ increasing, the time-distance in point $P_{x}$ would reduce, and the attention field intensity negative correlation with it would increase.

Based on the general driving behavior, the driver's concern degree has a more significant reduction from the front space to besides. And with the high speed, the driver's vision field width would be smaller, and pays less attention to the besides space. So in the fixed point $P_{y}$, the corresponding field intensity value would decrease with the target vehicle increasing.

Assume the two points $P_{x}$ and $P_{y}$ are on the same equipotential line. After determining the field intensity value of the equipotential line, as the velocity increasing, the point $P_{x}$ in this equipotential line would move forward, and the point $P_{y}$ would move toward the target vehicle, from the shape as Figure 4 shows. Obviously, with the speed getting big, the equipotential line shape would get sharp; if the speed is low, the equipotential line shape would get relatively round.

Meanwhile, like the field physics, the equipotential lines in attention field should have a nonoverlapping feature. It could assume that the ration between the $O P_{x}$ and $O P_{y}$ is fixed. $O P_{x}$ is the distance from intersection between the target vehicle driver's attention equipotential line and the vehicle traveling direction to vehicle $n . O P_{y}$ is the distance from the vehicle $n$ position to the intersection between the attention equipotential line and the direction vertical the vehicle traveling. Let the ratio be $\alpha$. The $\alpha$ is associated with the target vehicle speed, and also associated with the driver's own property. Clearly, based on the above analysis and Figure 4, for the same type of driver, the higher the speed, the smaller the $\alpha$.

The drivers could be divided into three types: cautious, aggressive, and balanced. Different types of drivers have different perceptions of the surrounding environment. It is embodied in the different-value $v_{\varepsilon}$ in formula (1). The cautious driver correspond to a relatively bigger $v_{\varepsilon}$, the aggressive type correspond to a smaller $v_{\varepsilon}$, and the $v_{\varepsilon}$ value of balanced driver is in the middle.

In Figure 4, the angle $\theta$ of the equipotential line in the first quadrant of the coordinate system with the vehicle $n$ as origin increases with point $P$ getting close to the target vehicle $n$ and becomes max at point $P_{x}$. The field intensity of

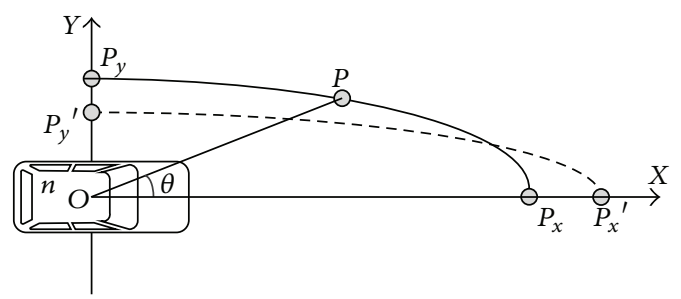

Figure 4: Attention field equipotential line trend schematic.

this equipotential line is determined by the velocity of vehicle $n$ and the length of $O P_{x}$. Let the length of $O P_{x}$ be $L_{0}$. And the mathematical expression of the corresponding equipotential line is shown as

$$
\rho=L_{0}-(1-\alpha) L_{0}|\sin \theta|
$$

The $P$ is an arbitrary point in equipotential line. Point $O$ is the position of vehicle $n$. The $\rho$ is distance between the point $P$ and the vehicle n. $\theta$ is the angle between the $O P$ and the direction (the $x$-axis in Figure 4 ) of vehicle $n$ traveling. $\alpha$ is the ratio between $O P_{x}$ and $O P_{y}$; its value is related to the speed of vehicle $n$. The $L_{0}$ is the distance between the target and the point $P_{x}$. It is related to the speed of vehicle $n$ and the corresponding field intensity of equipotential line.

2.2.2. The Field Intensity Modeling. It can obtain the formula (3) from (2):

$$
L_{0}=\frac{\rho}{1-(1-\alpha)|\sin \theta|} .
$$

For any point $(x, y)$ in the space like Figure 4 , the length $\rho_{(x, y)}$ between the origin (vehicle $n$ position) and it can be easily obtained. The parameter $\alpha$ is only related to the target vehicle speed. So the parameter $L_{0}$ could be obtained. Then combined with the formula (1), calculate the field intensity in point $(x, y)$. Finally, obtain the method to calculate the attention field intensity of arbitrary point in the space, as follows:

$$
E b_{n}(x, y)=\frac{v_{n}+v_{\varepsilon}}{\rho_{(x, y)}}\left[1-\left(1-\alpha\left(v_{n}\right)\right)\left|\sin \theta_{(x, y)}\right|\right] .
$$

2.2.3. Model frame. The driving behavior model should include two parts: one is the driver's attention field description, and the other is the attribute information and motion state of objects in the field. Similar to the physical field, the driving behavior based on the attention field model framework follows:

$$
F_{n}=E b_{n} \cdot g_{n-1}\left(v_{n-1}, v_{n}\right) .
$$

The $n-1$ is vehicle in the target vehicle $n$ driver's attention field is as in Figure 1. $v_{n-1}$ and $v_{n}$ are the velocity of vehicles $n$ and $n-1 . g_{n-1}$ is the characteristics of vehicle $n-1$ itself; it is relative to the relative speed and direction. $F_{n}$ is the effect of vehicle $n$. 
Form the visual point of view, the changing of $n-1$ vehicle position should affect the vehicle $n$ driver, but it is not directly reflected in the model frame. This is because this fact is fully taken into account in calculating the attention field intensity.

The $F_{n}$ in the modeling process usually consider setting it as the acceleration of target vehicle. And it is closely connected to the force affect the object in physical can directly reflect the attention effects.

\section{Model Analysis}

3.1. Parameters Analysis. In the attention field, the $\sin \theta_{(x, y)}$ and $\rho_{(x, y)}$ in formula (4) determine the space position; $v_{n}$ is the velocity of the vehicle. The $v_{\varepsilon}()$ is used to distinguish the different characteristics of the driver's attention field and describe the effect from the vehicle movement on the driver.

$v_{\varepsilon}()$ is affected by two factors: one is the driver's personality characteristics, the cautious driver corresponding to relatively big $v_{\varepsilon}$, and the aggressive driver corresponding to small $v_{\varepsilon}$. And the other factor is changing of the vehicle movement state. When the vehicle is in the accelerated state, the focus on the objects would be strengthened. It is shown as the field intensity of same position in attention field would increase, and the $v_{\varepsilon}$ becomes bigger. If the vehicle is decelerating, the attention to the front would reduce, and the $v_{\varepsilon}$ becomes smaller. In these two factors, the driver's personality characteristics should play a major role, while the status of vehicle is running relative to a supporting role.

The $v_{\varepsilon}$ is shown as formula (6); the $\varphi_{1}$ and $\varphi_{2}$ undetermined coefficient. $D_{n}$ is the membership function of driver personality characteristics. Its ranging is $D_{n} \in[0,1], D_{n}$ is increasing with the driver's personality aggressiveness increasing. When $D_{n}$ (driver) $=1$, it means the driver is extreme caution; if $D_{n}$ (driver) $=0$ it means the driver is extremely reckless:

$$
v_{\varepsilon}=\varphi_{1} e^{D_{n}(\text { driver })}+\varphi_{2}\left(e^{A_{n}\left(a_{n}, a_{\text {acc_max }}, a_{\text {dec_max }}\right)}-1\right) .
$$

$A_{n}$ is the ratio between the current acceleration and the maximum acceleration; it is shown as formula (7). The $a_{n}$ is the current acceleration of the vehicle; it is a sign scalar. $a_{\mathrm{acc} \text { max }}$ and $a_{\mathrm{dec} \text { max }}$ are the maximum acceleration and deceleration; they are the positive scalar:

$$
A_{n}\left(a_{n}, a_{\mathrm{acc} \_ \text {max }}, a_{\mathrm{dec} \_ \text {max }}\right)= \begin{cases}\frac{a_{n}}{a_{\mathrm{acc} \_\max }} & a_{n}>0, \\ 0 & a_{n}=0, \\ \frac{a_{n}}{a_{\mathrm{dec} \_\max }} & a_{n}<0 .\end{cases}
$$

The range of $A_{n}$ is $A_{n} \in[-1,1]$. By the characteristics of the exponential function, $e^{D_{n}}$ and $e^{A_{n}}-1$ are all the monotonically increasing functions as Figure 5 shows. When the character gets close to reckless driver, the value of $e^{D_{n}}$ is increasing, and the changing amplitude is also increased. It corresponds to the aggressive driver having a low degree concern for the obstructions, and the cautious driver is tense to deal with the objects in front.

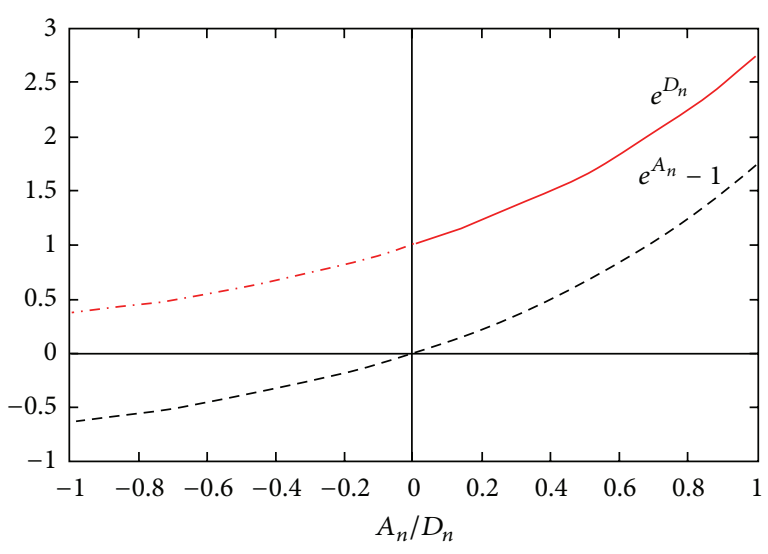

Figure 5: The value changing of $e^{D_{n}}$ and $e^{A_{n}}-1$.

The $\alpha\left(v_{n}\right)$, in formula (4), it value increases monotonically with decreasing speed. $\alpha\left(v_{n}\right)$ is defined as the formula (8). The $v_{\max }$ is the maximum speed the vehicle could reach. The $\alpha_{\max }$ is the value of $\alpha$ when the vehicle is stopping. And $\alpha_{\min }$ is the value of $\alpha$ when the vehicle reaches the maximum speed:

$$
\alpha\left(v_{n}\right)=\frac{\alpha_{\min }-\alpha_{\max }}{v_{\max }} v_{n}+\alpha_{\max } .
$$

The final attention field intensity is as follows; the $\gamma_{1}$ is adjustment factor:

$$
E_{n}=\gamma_{1} \cdot E b_{n}
$$

\subsection{The Improved Model}

3.2.1. The Improved Field Intensity of Objects. The model previously established can describe the attention field distribution. Because there are size differences of the objects in field, the field intensity of objects space is hard to represent with field strength at a point. It considers using the first curve integral to solve this problem.

The first form curvilinear integral is a commonly math method. Let $L$ be a smooth curve in plane $x O y$. The function $f(x, y)$ is bounded in $L$. The integral of the function on curve $L$ is $\int_{L} f(x, y) d s$.

For the driver of vehicle $n$ in Figure 6, limited by the vision, without considering the object height, in Figure 6, the $n$ vehicle driver's visual range of vehicle $n-1$ are $Q_{1} Q_{2}$ and $Q_{2} Q_{3}$. So we could simplify the $n-1$ vehicle with complicated shape to two segments $Q_{1} Q_{2}$ and $Q_{2} Q_{3}$. Then apply the first form curvilinear integral method to calculate the attention field intensity of the vehicle $n-1$.

However, the objects in the attention would be diverse and irregular, causing hard calculation of the first form curvilinear integral. Consider using the projection of the object borders in the driver's visual range to represent the object's position. Then calculate the field intensity with the first form curvilinear integral of this projection; the result is the object's field intensity, such as the vehicle $n-1$ in Figure 6 . 


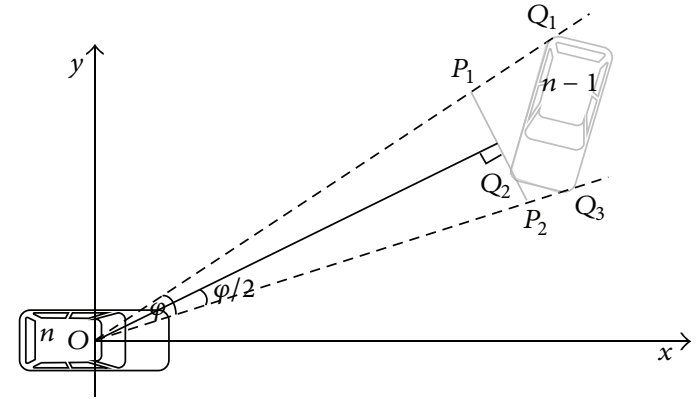

FIGURE 6: Object projection in driver's visual range schematic I.

The projection line $P_{1} P_{2}$ is determined by angle $\angle Q_{1} O Q_{3}$ and the nearest point $Q_{2}$ to the driver on object vehicle $n-1$. Let $\angle Q_{1} O Q_{3}$ value be $\varphi$, and the line passing through the point $Q_{2}$ and vertical to the angular bisector of $\angle Q_{1} O Q_{3}$ has two intersection points with $O Q_{1}$ and $O Q_{2}$ which are $P_{1}$ and $P_{2}$.

Assume the coordinate of $P_{1}, P_{2}$ are $\left(x_{1}, y_{1}\right)$ and $\left(x_{2}, y_{2}\right)$, and let $x_{1}<x_{2}$. When the segment $P_{1} P_{2}$ is not perpendicular to the traveling direction (the $x$-axis in Figure 6), the linear equation is as follows:

$$
y=\frac{y_{2}-y_{1}}{x_{2}-x_{1}}\left(x-x_{1}\right)+y_{1} .
$$

Let

$$
x=t, \quad y=\frac{y_{2}-y_{1}}{x_{2}-x_{1}} t+\frac{y_{1} x_{2}-y_{2} x_{1}}{x_{2}-x_{1}} .
$$

Then, the curve integral of the segment in driver's attention field is shown in formula (12). The $E_{n}(x, y)$ is the field intensity model in formula (4):

$$
\begin{aligned}
E_{n}(n-1) & =\int_{P_{1} P_{2}} E_{n}(x, y) d s \\
& =\int_{x_{1}}^{x_{2}} E_{n}\left(t, \frac{y_{2}-y_{1}}{x_{2}-x_{1}}\left(t-x_{1}\right)+y_{1}\right) \\
& \cdot \sqrt{1+\left(\frac{y_{2}-y_{1}}{x_{2}-x_{1}}\right)^{2}} d t .
\end{aligned}
$$

Let $\left(y_{2}-y_{1}\right) /\left(x_{2}-x_{1}\right)=k,\left(y_{1} x_{2}-y_{2} x_{1}\right) /\left(x_{2}-x_{1}\right)=$ $b$, and when the $P_{1}, P_{2}$ are both above the $x$-axis shown in Figure 6, formula (12) could be simplified as follows:

$$
\begin{aligned}
E_{n}(n-1)= & \left(v_{n}+v_{\varepsilon}\right) \sqrt{k^{2}+1} \\
& \times\left\{\int_{x_{1}}^{x_{2}} \frac{1}{\sqrt{t^{2}+(k t+b)^{2}}} d t\right. \\
& \left.+(1-\alpha) \int_{x_{1}}^{x_{2}} \frac{k t+b}{t^{2}+(k t+b)^{2}} d t\right\} .
\end{aligned}
$$

If the points $P_{1}$ and $P_{2}$ are below the $x$-axis shown in Figure 6, we could get formula (14)

$$
\begin{aligned}
E_{n}(n-1)= & \left(v_{n}+v_{\varepsilon}\right) \sqrt{k^{2}+1} \\
& \times\left\{\int_{x_{1}}^{x_{2}} \frac{1}{\sqrt{t^{2}+(k t+b)^{2}}} d t\right. \\
& \left.+(1-\alpha) \int_{x_{1}}^{x_{2}} \frac{-k t-b}{t^{2}+(k t+b)^{2}} d t\right\} .
\end{aligned}
$$

If the $P_{1}$ and $P_{2}$ are located across the $x$-axis as Figure 7 shows, assume the interaction point between $P_{1} P_{2}$ and $x$-axis is $P_{3}\left(x_{3}, 0\right)$, and let $x_{1}<x_{3}<x_{2}$. It is needed to calculate the field intensity of $P_{1} P_{3}$ and $P_{3} P_{2}$ separately, as follows:

$$
\begin{aligned}
E_{n}(n-1)=\left(v_{n}\right. & \left.+v_{\varepsilon}\right) \sqrt{k^{2}+1} \\
& \times\left\{\int_{x_{1}}^{x_{3}} \frac{1}{\sqrt{t^{2}+(k t+b)^{2}}} d t\right. \\
& +(1-\alpha) \int_{x_{1}}^{x_{3}} \frac{k t+b}{t^{2}+(k t+b)^{2}} d t \\
& +\int_{x_{3}}^{x_{2}} \frac{1}{\sqrt{t^{2}+(k t+b)^{2}}} d t \\
& \left.+(1-\alpha) \int_{x_{3}}^{x_{2}} \frac{-k t-b}{t^{2}+(k t+b)^{2}} d t\right\} .
\end{aligned}
$$

The prerequisite of the formula (15) is $x_{1}<x_{2}$. And if $x_{1}=x_{2}$, let $x_{1}=x_{2}=a$ and assume that

$$
x=a, \quad y=t .
$$

The field intensity of segment $P_{1} P_{2}$ is shown as follows:

$$
\begin{aligned}
\int_{P_{1} P_{2}} E_{n}(x, y) d s= & \left(v_{n}+v_{\varepsilon}\right) \sqrt{k^{2}+1} \\
& \times\left[\int_{y_{1}}^{y_{2}} \frac{1}{\sqrt{a^{2}+t^{2}}} d t+\int_{y_{1}}^{y_{2}} \frac{|t|}{a^{2}+t^{2}} d t\right] .
\end{aligned}
$$

And if the $P_{1}$ and $P_{2}$ are located across the $x$-axis as Figure 7 shows, it is needed to calculated the field intensity of $P_{1} P_{3}$ and $P_{3} P_{2}$ separately, as follows:

$$
\begin{aligned}
\int_{P_{1} P_{2}} E_{n}(x, y) d s= & \left(v_{n}+v_{\varepsilon}\right) \sqrt{k^{2}+1} \\
& \times\left\{\int_{y_{1}}^{y_{3}} \frac{1}{\sqrt{a^{2}+t^{2}}} d t+\int_{y_{1}}^{y_{3}} \frac{t}{a^{2}+t^{2}} d t\right. \\
& \left.\quad+\int_{y_{3}}^{y_{2}} \frac{1}{\sqrt{a^{2}+t^{2}}} d t+\int_{y_{3}}^{y_{2}} \frac{-t}{a^{2}+t^{2}} d t\right\} .
\end{aligned}
$$




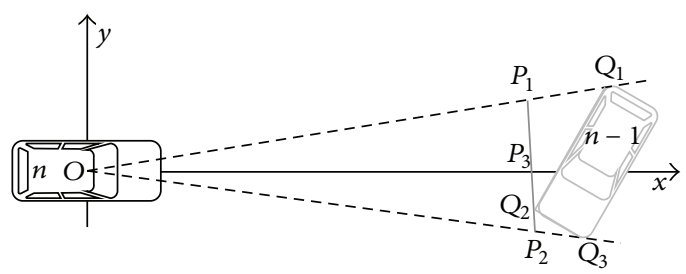

FIGURE 7: Object projection in driver's visual range schematic II.

Thus, it can extend this method to calculate any form of the object. Because the projection of the object could finally be attributed to a segment in attention field. And if the segment endpoints is known, it is easy to get the field intensity $E_{n}\left(P_{1} P_{2}\right)$ of the segment than getting that of the object.

3.2.2. The Attention Field Range. Limited by the human eye vision scope, the driver's attention field is finite. According to general cognitive, with the vehicle speed increasing, the driver's attention distance expands in the direction of travelling. But limited by the driver's visual capacity, the attention distance exits upper limit. Combined with previous, take the max value of upper limit to be 420 meter, and the min upper limit to be 50 meter. So assume the attention range in the traveling direction is shown as formula (19). The unit of $v_{n}$ is $\mathrm{km} / \mathrm{h} . R$ is the maximum visual attention distance with the speed $v_{n}$; its unit is meter:

$$
R= \begin{cases}60 & v_{n}<40 \\ 6 v_{n}-180 & v_{n} \in[40,100] \\ 420 & v_{n}>100\end{cases}
$$

The driver's visual range would be gradually narrow with the vehicle speed increasing. Assume the attention field range is shown as in Figure 8, the points $L_{l 1}, L_{l 2}, L_{r 1}$, and $L_{r 2}$ are the four boundary points of the attention field effect range. Let the angle between $L_{l 1} O$ and $L_{r 1} O$ be $\eta$. The relationship between $\eta$ and vehicle speed is shown in Table 1. According to the data in Table 1, establish the corresponding math relationship between the speed and the visual angle $\eta$ with the regression least squares method as follows:

$$
\eta=0.0064 v_{n}^{2}-1.8244 v_{n}+159.55 .
$$

In the transverse direction, the driver usually only focuses on the objects in the driveway and the adjacent two-lane and neglects the objects outside this range. Assume the length $L_{l 2} L_{r 2}$ in Figure 8 is $d_{y}$; let it equal to triple lane width as formula (21) shows. The $d_{\text {lane }}$ is the lane width. And the coordinates of the four boundary points in the attention field effect range could be obtained. And the attention field effect scope is identified:

$$
d_{y}=3 d_{\mathrm{lane}}
$$

3.2.3. Effect of Object Motion Direction. There would be different impacts of the objects in the same spatial position but
TABLE 1: Correspondence between speed and visual angle.

\begin{tabular}{lcccc}
\hline Vehicle speed $(\mathrm{km} / \mathrm{h})$ & 0 & 40 & 70 & 100 \\
\hline${\text { Visual angle }\left(\text { degree }^{\circ}\right)}^{\circ}$ & 160 & 95 & 65 & 40 \\
\hline
\end{tabular}

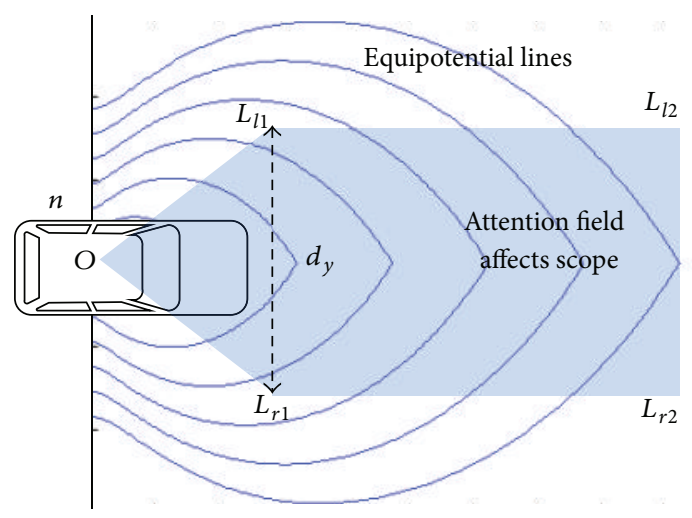

FIgURE 8: Attention field affects scope schematic.

with different relative moving direction to the target vehicle driver. In model, this embodies the field intensity changing.

In physical, the gradient describes the steepest changing direction of the field intensity. Because of the discontinuities of the attention field on the $x$-axis, using exhaustive method to calculate the direction with the field intensity greatest changing point is needed.

Take the specific point $(x, y)$ as the center, and the specific distance $r$ as the radius, calculate the field strength on the circumference. $r$ is positivly relative with the relative velocity. Compared with the field strength at point $(x, y)$, the connection between the $(x, y)$ and the biggest field intensity changing point is the direction of gradient in attention field; let it be $G a(x, y)$.

Meanwhile, we could get the angle between the $G a(x, y)$ and direction of the relative movement object speed; let it be angle $\varphi$. If the relative speed coincides with $G a(x, y)$, it indicates that the object would have a relatively big impact on driver. And with the angle $\varphi$ increasing, the psychological pressure of the driver from the object get weakened.

3.2.4. The Effect of Psychological Drive. Driving task brings the driver psychological pressure. In the free state, the pressure comes from the driver's desired speed. The driver would accelerate by adjusting the speed and finally stabilize nearby the expectation. In the car-following state, the drive force comes from the large driving distance to the leading car. In order to ensure traffic efficiency, the driver accelerates to maintain the proper headway or the distance to the leading vehicle. The psychological drive force and the psychological pressure from the attention field alternation affect the driver's driving behavior.

\subsection{Numerical Simulation}

3.3.1. The Free State. When the vehicle travels in the free state, the surrounding vehicles sparse; the driver's mainly 




FIGURE 9: Schematic of vehicle running track.

restrictions are road conditions and constraints from the traffic facilities. The lane marking and the road barrier are the most common driving traffic facilities. The road surface conditions determine the driver's desired speed, and lane markings and road barrier constrain the direction of the vehicle trajectory. Analyze the driving behavior in a free state on road as Figure 9 shows.

Take the impacts of driver from lane markings and road barrier as a simple vector superposition. Assume the vehicle running in the road as Figure 9 shows without any other vehicles and obstacles. Let the lane width be 3.5 meters; the distance between the vehicle and the road barrier in the perpendicular to the traveling direction is 1.5 meters at initial time. The initial velocity and the expected velocity are both $20 \mathrm{~m} / \mathrm{s}$.

Apply the model this paper proposed to simulate the vehicle running processing. The displacement curve is shown in Figure 9. In initial simulate time, the vehicle is close to the road barrier. For keeping the safety, the vehicle decelerated and shifted away from the road barrier in initial time stage. When the offset reached a certain extent, limited by the lane line in Figure 9, the vehicle stopped moving in the transverse direction. At this time, because the distance between the road barrier and the vehicle in vertical direction reached a safety extent, the driver maintained the vehicle running state.

In the two-lane road, according to the observed situation in traffic environment, the vehicle is not usually located in the middle of the lane, but has a certain extent offset in the vertical direction of road. This phenomenon is due to psychological stress caused by the road edge. In order to avoid this kind of pressure, the driver would leave away the road edge to reduce the attention to it if the road condition is allowed, thereby forming the above-described phenomenon.

3.3.2. The Waiting Pedestrian Interference. It is a normal phenomenon that the pedestrian crosses the road with no traffic signal controlling. As shown in Figure 10, the pedestrian waiting at roadside A for the gap of the traffic flow to cross the road reaches another roadside $B$. In this process, in addition to the crossing pedestrian affecting the vehicle in traffic flow, the ones in the waiting area also impact the driver. Then achieve the numerical simulation of this phenomenon.

The waiting pedestrians mostly would be cluster. For the cluster pedestrians, their projection in the driver's attention field is shown as the line $Q_{1} Q_{2}$ in Figure 11. Based on the

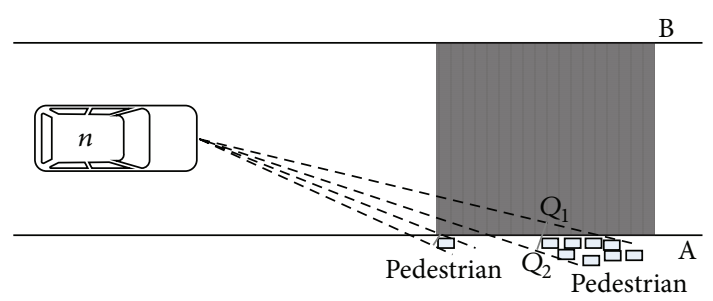

Figure 10: Pedestrian crossing schematic.

attention field introduction, the effect of the cluster pedestrian relative to the area and shape of cluster pedestrian. Assume the individual pedestrian occupies $0.5 * 0.5 \mathrm{~m}^{2}$ space. Let the number of the pedestrians be 30 , array in $10 \times 3$ matrix; the first row of the crow is at the roadside A as Figure 10 shows. Let the running vehicles queue number be 50 . The first vehicle of the queue has a $50 \mathrm{~m}$ distance to the pedestrians. The initial and expected velocities are both $20 \mathrm{~m} / \mathrm{s}$. The space headway in the queue is $40 \mathrm{~m}$.

The vehicle would slow down when it gets close to the pedestrian. Because the vehicle of the queue is in the carfollowing state, the decelerate behavior would spread back in the queue, causing the whole velocity of the traffic flow to reduce. And when the vehicle passe the pedestrians, it would be affected by both the leading vehicle and the pedestrians, if the leading just pass the pedestrian effect range and begin to accelerate, the following vehicle driver would not be able to speed up with the leading as usual, but continue to bes affected by the pedestrian. Until leaving the pedestrian effect range, the vehicle accelerates to the expected speed with the psychological driving force. So in Figure 11(a), the velocity of the vehicles queue would first decrease and then recover; the density of the queue declines continuously after the initial slight increase. And maintains stable after leaving the effected range.

It could be seen from Figure 11(b) that, after the vehicles queue passing the pedestrian effect range, the queue would be stretched. This is because the first vehicle passes the pedestrians effect range with reducing the speed, the slowdown state spread back to the queue. And when the first vehicle just passed the effect range, the expected speed drives the driver to accelerate to the initial state. But this state would not spread immediately because the subsequent vehicles are still affected by the pedestrian. The space headway of the neighboring vehicles increases. And limited by the expected velocity, even the subsequent vehicles have passed the effect range, they would not accelerate continuously but maintain an expected speed. So if the smooth running traffic flow passes through the pedestrian effect area, the spacing between the neighboring vehicles and the density of the traffic flow decrease, causing the number of passing vehicles in unit time to reduce, and the traffic capacity of the road reduces.

\section{Conclusion}

This paper presents a new method of modeling the driving behavior. Used the field concepts in physical to establish 


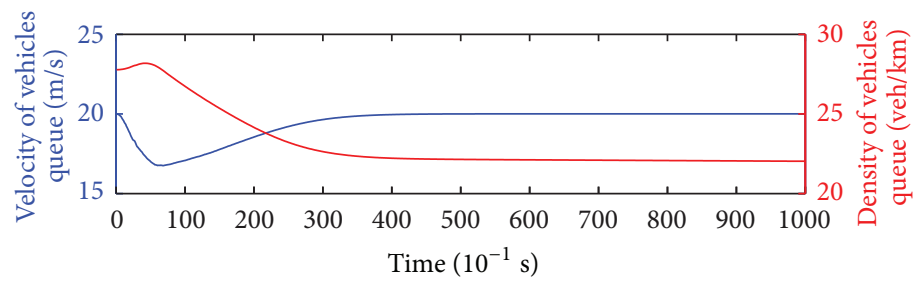

(a) Velocity-density changing of vehicles queue

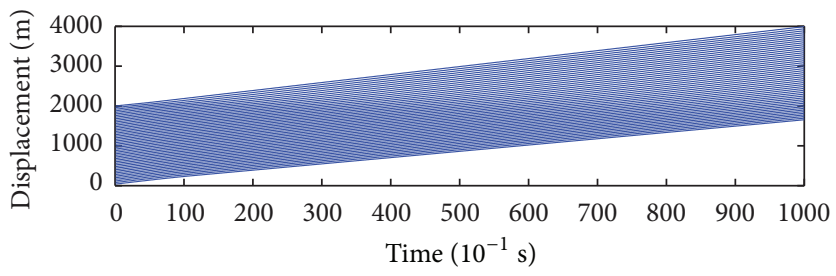

(b) Vehicles running track

FIGURE 11: Displacement of the vehicles in queue.

a driver's attention field to describe the distribution of the attention. The result shows that the model can simulate the operation of driver process, and in line with the individuals vehicle movement and the traffic flow running.

The model extends the scope of the driving behavior model, getting rid of the limited by car-following behavior. And the model could output the vehicle in the lateral movement. Meanwhile, because of the complex of the driving behavior, the model is not capable of describing all driving behaviors. The model structure needs to be further restructured and strengthened. And more experimental data is needed to specificly calibrate the parameters to make the model applied in practice.

\section{Conflict of Interests}

The author declare that there is no conflict of interests regarding the publication of this paper.

\section{Acknowledgment}

This work is supported by the National Science Foundation of China (nos. 51278220, 51278454, and 51108208).

\section{References}

[1] M. Brackstone and M. McDonald, "Car-following: a historical review," Transportation Research F, vol. 2, no. 4, pp. 181-196, 1999.

[2] A. Kesting and M. Treiber, "Calibrating car-following models by using trajectory data methodological study," Transportation Research Record, no. 2088, pp. 148-156, 2008.

[3] Y. Hollander and R. Liu, "The principles of calibrating traffic microsimulation models," Transportation, vol. 35, no. 3, pp. 347362, 2008.

[4] D. C. Gazis, R. Herman, and R. W. Rothery, "Nonlinear follow the leader models of traffic flow," Operations Research, vol. 9, no. 4, pp. 913-933, 1961.
[5] S. Jin, D.-H. Wang, C. Xu, and Z.-Y. Huang, "Staggered carfollowing induced by lateral separation effects in traffic flow," Physics Letters A, vol. 376, no. 3, pp. 153-157, 2012.

[6] B. Gunay, "Car following theory with lateral discomfort," Transportation Research B, vol. 41, no. 7, pp. 722-735, 2007.

[7] S. Jin, D. Wang, P. Tao, and P. Li, "Non-lane-based full velocity difference car following model," Physica A, vol. 389, no. 21, pp. 4654-4662, 2010.

[8] H. B. Zhu and S. Q. Dai, "Analysis of car-following model considering driver's physical delay in sensing headway," Physica A, vol. 387, no. 13, pp. 3290-3298, 2008.

[9] S. Ossen and S. P. Hoogendoorn, "Heterogeneity in car-following behavior: theory and empirics," Transportation Research C, vol. 19, no. 2, pp. 182-195, 2011.

[10] H. Rakha and M. Arafeh, "Calibrating steady-state traffic stream and car-following models using loop detector data," Transportation Science, vol. 44, no. 2, pp. 151-168, 2010.

[11] R. Hoogendoorn, S. Hoogendoorn, K. Brookhuis, and W. Daamen, "Psychological elements in car-following models: mental workload in case of incidents in the other driving lane," Procedia Engineering, vol. 3, no. 1, pp. 87-99, 2010.

[12] P. Wagner, "Analyzing fluctuations in car-following," Transportation Research B, vol. 46, no. 10, pp. 1384-1392, 2012.

[13] F. E. Gunawan, "Two-vehicle dynamics of the car-following models on realistic driving condition," Journal of Transportation Systems Engineering and Information Technology, vol. 12, no. 2, pp. 67-75, 2012.

[14] W. Wang, K. Bengler, and G. Wets, "Discrete dynamics in transportation system," Discrete Dynamics in Nature and Society, vol. 2013, Article ID 234970, 2 pages, 2013.

[15] H. Guo, W. Wang, W. Guo, and F. Zhao, "Modeling lane-keeping behavior of bicyclists using survival analysis approach," Discrete Dynamics in Nature and Society, vol. 2013, Article ID 197518, 6 pages, 2013. 


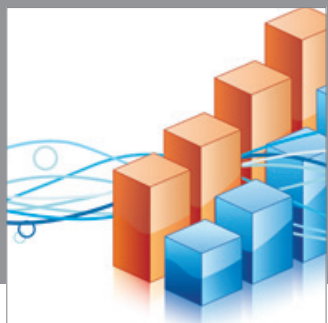

Advances in

Operations Research

mansans

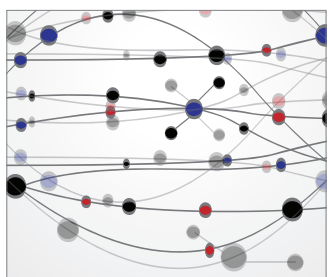

The Scientific World Journal
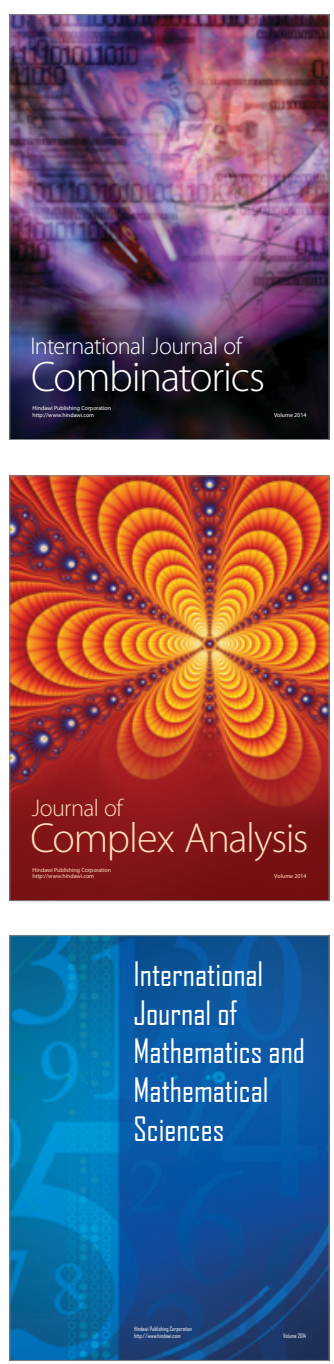
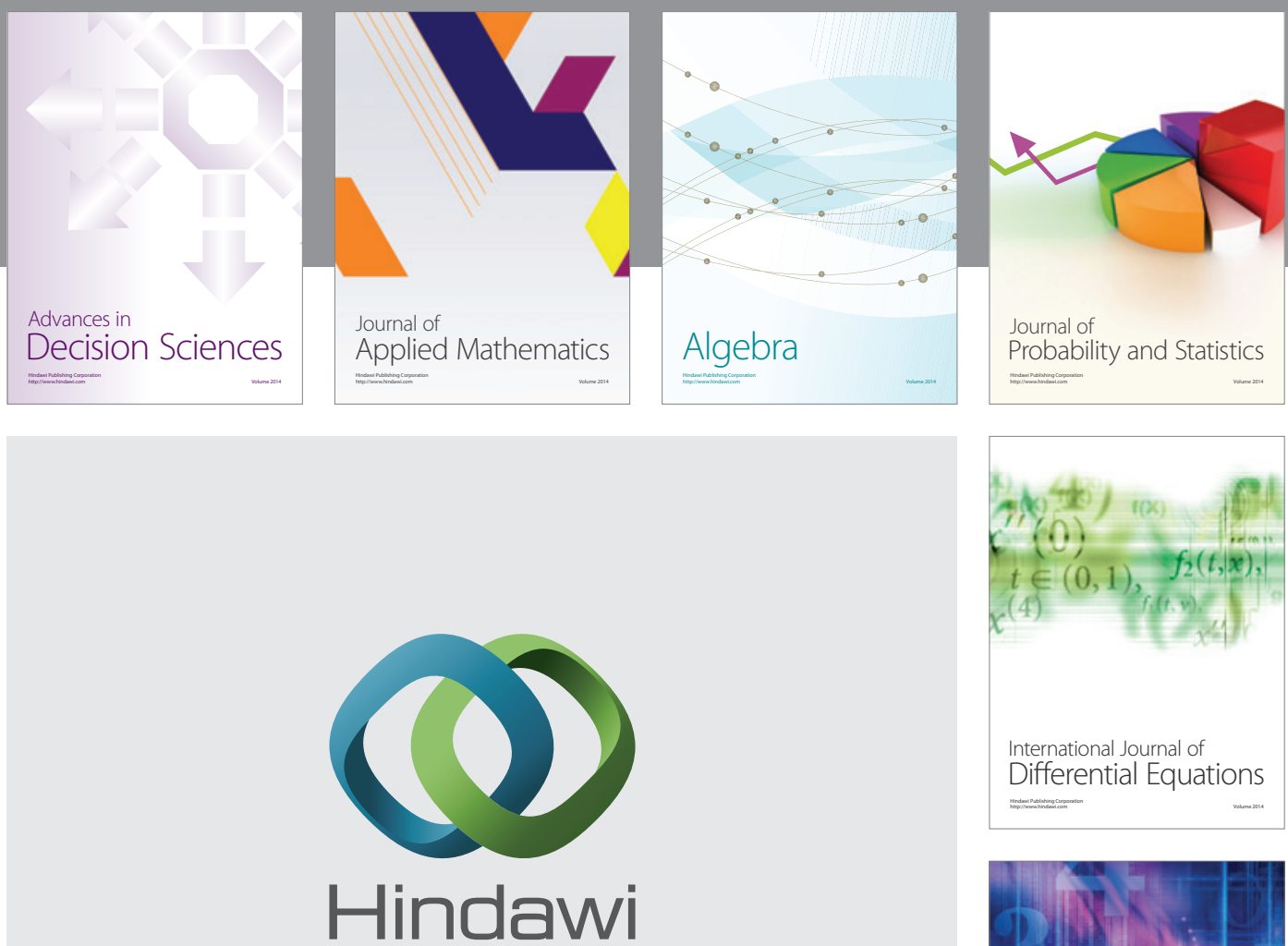

Submit your manuscripts at http://www.hindawi.com
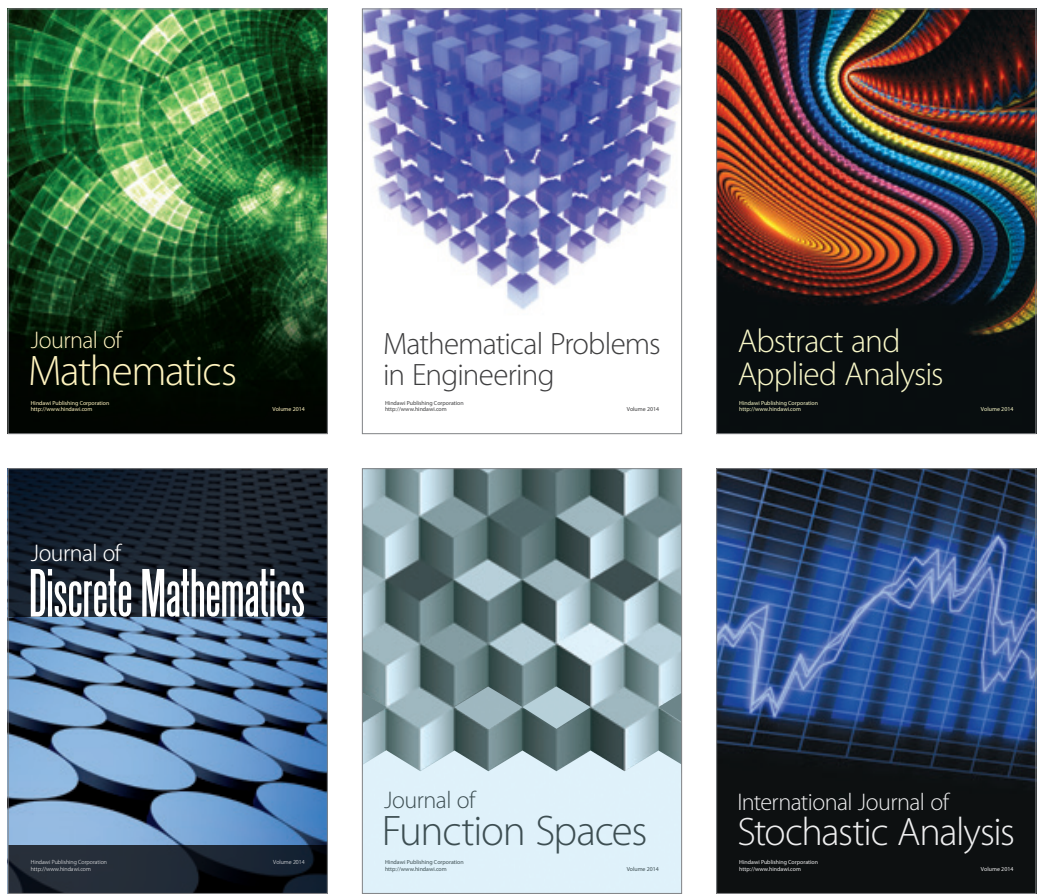

Journal of

Function Spaces

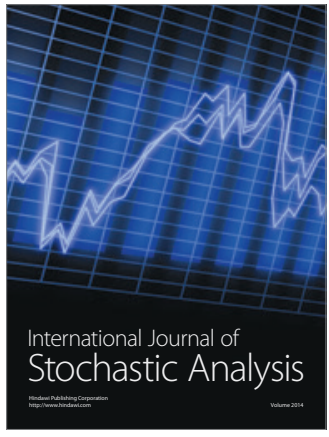

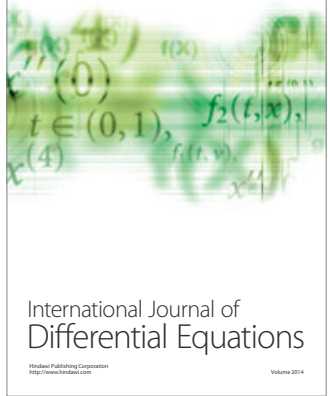
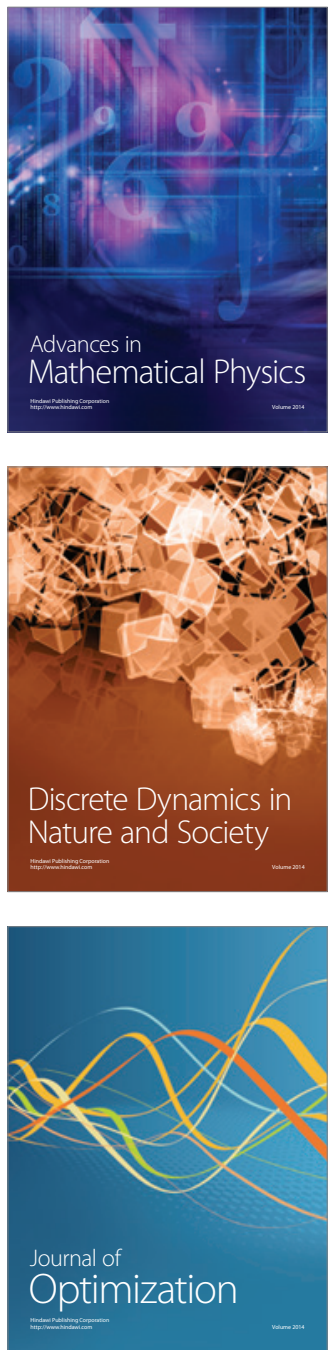